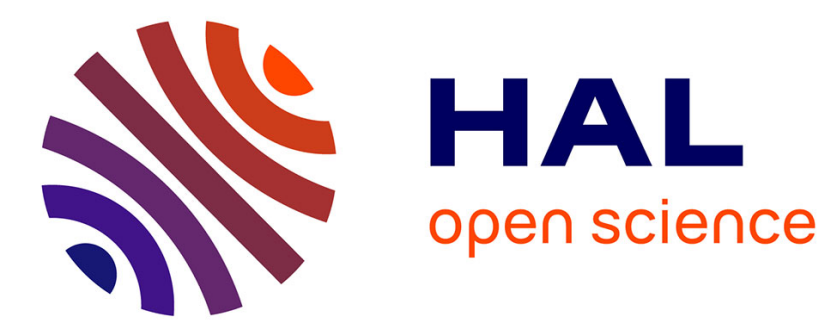

\title{
Effets de taille et interactions chimiques
}

Annie Blandin, J.L. Deplanté

\section{To cite this version:}

Annie Blandin, J.L. Deplanté. Effets de taille et interactions chimiques. J. Phys. Radium, 1962, 23

(10), pp.609-612. 10.1051/jphysrad:019620023010060900 . jpa-00236647

\section{HAL Id: jpa-00236647 https://hal.science/jpa-00236647}

Submitted on 1 Jan 1962

HAL is a multi-disciplinary open access archive for the deposit and dissemination of scientific research documents, whether they are published or not. The documents may come from teaching and research institutions in France or abroad, or from public or private research centers.
L'archive ouverte pluridisciplinaire HAL, est destinée au dépôt et à la diffusion de documents scientifiques de niveau recherche, publiés ou non, émanant des établissements d'enseignement et de recherche français ou étrangers, des laboratoires publics ou privés. 


\title{
EFFETS DE TAILLE ET INTERACTIONS CHIMIQUES
}

\author{
Par A. BLANDIN et J. L. DEPLANTÉ, \\ Service de Physique des Solides, B. P. 11, Faculté des Sciences d’Orsay, Seine-et-Oise, France.
}

\begin{abstract}
Résumé. - L'étude de la structure électronique des défauts dans les métaux met en évidence l'existence d'oscillations de la densité électronique et du potentiel autour de chaque impureté. Ce modèle est appliqué à quelques propriétés dues aux impuretés et aux lacunes dans les métaux et alliages : interactions chimiques, effets de taille et variation de compressibilité. Les effets prévus dépendent essentiellement de la valence de la matrice. Les résultats expérimentaux pour les métaux monovalents (métaux nobles et alcalins) divalents ( $\mathrm{Mg}, \mathrm{Zn}, \mathrm{Cd})$ et trivalents (Al) sont en bon accord avec la théorie.
\end{abstract}

Abstract. - The study of the electronic structure of defects in metals shows the existence of oscillations in the electronic density and electric potential around each impurity. This model is applied to some properties of metals and alloys viz chemical energy of interaction, size effect and compressibilities. It is predicted that these properties depend mainly on the valency of the matrix. Experimental results are discussed in the cases of the monovalent metals (noble and alkali metals) divalent metals $(\mathrm{Mg}, \mathrm{Zn}, \mathrm{Cd})$ and trivalent aluminium. They are in good agreement with the theory.

La structure électronique des défauts (impuretés ou lacunes) dans les métaux normaux commence à être bien comprise : en plus d'un écran à courte distance, il existe des oscillations de densité électronique autour de chaque impureté [1], se comportant à grande distance comme

$$
\cos \left(2 k_{F} r+\varphi\right) / r^{3}
$$

où $k_{F}$ est le vecteur d'onde de Fermi.

Dans cette communication, le modèle est appliqué à quelques propriétés des cristaux et alliages : interaction entre défauts, effets de taille et variation de compressibilité dus aux défauts. Dans une première partie, on rappelle la structure électronique d'une impureté et on discute les différents cas possibles : impuretés de valence différente, de période différente, lacunes. La seconde partie montre comment déduire les propriétés des défauts à partir de la répartition électrostatique autour de chaque défaut. Dans la troisième partie, on compare les résultats théoriques et expérimentaux.

1. Structure électronique des défauts. - Le premier type de défaut a été étudié par la méthode de Thomas-Fermi. L'effet d'une impureté de différence de valence $z$ avec la matrice et de son écran est représenté par un potentiel à un électron $z \mathrm{e}^{-\lambda r / r}$. On a montré depuis qu'il existe de plus à grande distance des oscillations de densité électronique et de potentiel [1]. Pour un gaz d'électrons libres perturbés par une charge $z$, les changements de densité électronique $\Delta \rho(r)$, de potentiel électrique $V(r)$ et de champ électrique $E(r)$ ont la forme asymptotique.

$$
\begin{aligned}
\Delta \rho(r) & \simeq-\frac{a}{r^{3}} \cos \left(2 k_{F} r+\varphi\right) \\
V(r) & \simeq \frac{a \pi}{k_{F}^{2} r^{3}} \cos \left(2 k_{F} r+\varphi\right) \\
E(r) & \simeq \frac{a 2 \pi}{k_{F} r^{3}} \sin \left(2 k_{F} r+\varphi\right)
\end{aligned}
$$

où $k_{F}$ est le vecteur d'onde de Fermi ; l'amplitude $a$ des oscillations est pratiquement proportionnelle à $z$ et $\varphi$ est un facteur de phase.

Dans le cas où $z$ est petit, on peut calculer exactement le potentiel self-consistant de Hartree [2] décrivant la charge $z$ et son écran

$$
V(r)=\frac{z}{2 \pi^{2}} \int \frac{\mathrm{e}^{i K \cdot r} \mathrm{~d}^{3} K}{K^{2}+\frac{2 k_{F}}{\pi} g(K)}
$$

où

$$
g(K)=1+\frac{k_{F}}{K}\left(1-\frac{K^{2}}{4 k_{F}^{2}}\right) \log \left|\frac{K+2 k_{F}}{K-2 k_{F}}\right|
$$

avec

$$
\Delta \rho(r)=\frac{k_{F}}{2 \pi^{2}} \int V(K) g(K) \mathrm{e}^{i K \cdot r \mathrm{~d}^{3} K}
$$

Ceci revient à décrire le gaz d'électrons libres par une constante diélectrique $\varepsilon(K)$ à fréquence nulle dépendant du vecteur d'onde $K$ :

$$
\varepsilon(K)=1+\frac{2 k_{F}}{\pi K^{2}} g(K)
$$

à partir de (2) et (4) on peut obtenir les formes asymptotiques à grandes distances de $\Delta \rho(r)$ et $V(r)$ 
où les oscillations sont dues à la singularité en $\left(K-2 k_{F}\right) \log \left|K-2 k_{F}\right| \operatorname{de} g(K)$

$$
\begin{gathered}
\Delta \rho(r) \simeq-\frac{z k_{F}}{\left(2 \pi k_{F}+1\right)^{2}} \frac{\cos 2 k_{F} r}{r^{3}} \\
V(r) \simeq \frac{z \pi}{k_{F}\left(2 \pi k_{F}+1\right)^{2}} \frac{\cos 2 k_{F} r}{r^{3}}
\end{gathered}
$$

Les figures 1 et 2 comparent les résultats obtenus pour $\Delta \rho(r)$ et $V(r):(3)$ et (2) et leurs formes asymptotiques (5); le potentiel de Thomas-Fermi est

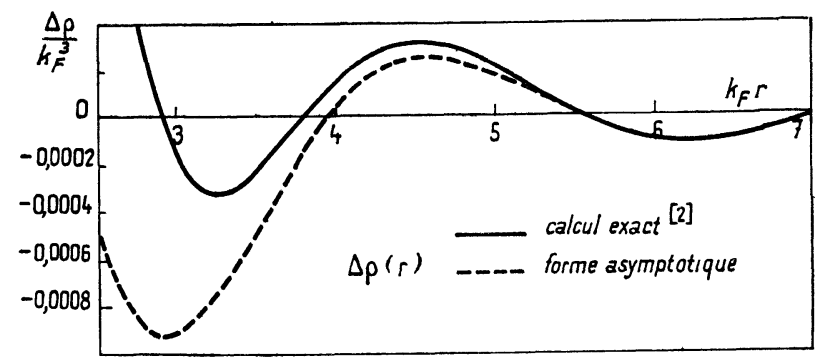

Frg. 1.

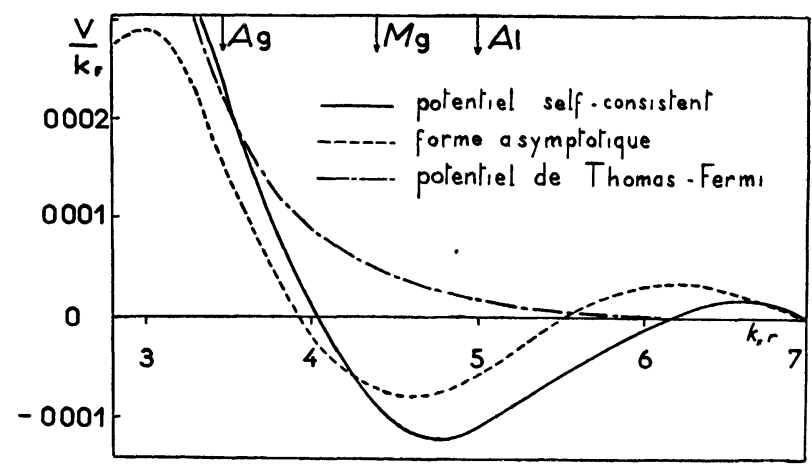

FIG. 2.

également reproduit sur la figure 2. (Ces résultats sont calculés pour une densité électronique donnant un rayon $r_{s}$ de sphère électronique $r_{s}=3 \mathrm{U}$. A.) Sur les figures 1 et 2 , on peut voir également les positions des atomes premiers voisins dans le cas des métaux à structure compacte monovalents $(\mathrm{Ag})$ divalents $(\mathrm{Mg})$ et trivalents (Al). Le signe de $\Delta \rho(r)$ et $V(r)$ sur les sites premiers voisins dépend donc uniquement de la valence de la matrice. En particulier, $V(r)$ change de signe en passant des métaux monovalents aux métaux polyvalents. Des conclusions semblables sont valables pour les champs électriques et les gradients de champs électriques sur les premiers voisins. Les figures 1 et 2 montrent d'autre part que les formes asymptotiques (5) sont de bonnes approximations même à assez courte distance. (Pour les cristaux monovalents, la valeur de $V(r)$ n'est pas très différente de la valeur calculée par la méthode de Thomas-Fermi ; ceci explique quelques succès obtenus avec cette méthode. Mars, il faut remarquer quela courbure a le mauvais signe).
Les résultats obtenus avec des électıons libres peuvent être généralisés aux électrons de Bloch pour tout type de défaut [3], [4]. Dans le cas d'une bande de conduction à surfaces d'énergie constante sphériques, les résultats sont analogues à (1), mais l'amplitude des oscillations est multipliée par un facteur dépendant uniquement de la matrice [4]. Ce facteur diffère suivant que l'on étudie le potentiel, champ électrique ou gradient de champ. Il est de l'ordre de l'unité pour les problèmes étudiés dans cette communication [4]. Dans ce qui suit, on utilisera donc la forme asymptotique de $V(r)$ :

$$
V(r) \simeq \frac{\alpha \pi}{k_{F}\left(2 \pi k_{F}+1\right)^{2}} \frac{\cos 2 k_{F} r}{r^{3}}
$$

on prend donc le facteur de phase $\varphi$ égal à zéro, ce qui n'est valable que pour d'assez petites perturbations et le facteur dû aux électrons de Bloch égal à un. L'amplitude $\alpha$ est déterminée ainsi :

a) dans le cas d'une différence de valence $z$ entre la matrice et l'impureté $\alpha=z$;

$b$ ) dans le cas d'une différence de période ( $\mathrm{K}$ dans Rb par exemple), $\alpha$ est calculée à l'aide d'ondes planes orthogonalisées et de pseudopotentiels [5] ;

c) dans le cas d'une lacune $\alpha=-z_{0}, z_{0}$ étant la valence de la matrice.

La formule (6) met clairement en évidence l'influence de la valence de la matrice sur la valeur de $V(r)$. En particulier $V(r)$ pour les premiers voisins d'un défaut est positif pour les matrices monovalentes, négatif pour les matrices di et trivalentes.

2. Quelques propriétés des défauts. - L'influence des changements de densité électronique dus aux défauts est maintenant étudiée dans quelques cas :

a) Energie chimique d'interaction. - Soit deux charges $z_{1}$ et $z_{2}$ dans un gaz d'électrons libres. L'énergie d'interaction $W(r)$ entre les deux charges distantes de $r$, est alors :

$$
W(r)=\frac{z_{1} z_{2}}{2 \pi^{2}} \int \frac{\mathrm{e}^{i K \cdot r}}{K^{2} \varepsilon(K)} \mathrm{d}^{3} K
$$

où $\varepsilon(K)$ est la constante diélectrique $\varepsilon(K)$ à fréquence nulle, pour le vecteur d'onde $K$. Dans l'approximation de Hartree self-consistante, $\varepsilon(K)$ a la valeur donnée par (4) d'où l'on déduit $W(r)$ [6] et sa forme asymptotique :

$$
W(r) \simeq \frac{z_{1} z_{2} \pi}{k_{F}\left(2 \pi k_{F}+1\right)^{2}} \frac{\cos 2 k_{F} r}{r^{3}}
$$

Pour deux défauts quelconques caractérisés par les amplitudes de leurs oscillations $\alpha_{1}$ et $\alpha_{2}$. On peut montrer qu'il suffit de remplacer $z_{1} z_{2}$ par $\alpha_{1} \alpha_{2}$. Dans le calcul de l'énergie d'interaction dans un alliage dilué, par exemple, on ne tiendra compte que des interactions entre proches voisins qui sont les plus importantes. 
b) Effets de taille et compressibilités. - On utilise le modèle suivant : $\mathrm{Si} z_{0}$ est la valence de la matrice la force $F(r)$ exercée sur chaque ion à la distance $r$ du défaut est radiale : $F(r)=-z_{0} \partial V / \partial r$. En ne considérant que l'influence d'un défaut sur les ions premiers voisins à la distance $r_{0}$, on remplace la force $F\left(r_{0}\right)$ sur les $n$ voisins par une pression $p=n F\left(r_{0}\right) / 4 \pi r_{0}^{2}$ exercée sur la sphère de rayon $r_{0}$ - le milieu est isotrope à la compressibilité $\chi$ de la matrice et le défaut est représenté par une sphère de rayon $r_{a}$ ( $r_{a}$ rayon atomique de la matrice) et de compressibilité $\chi^{\prime}$.

Le changement relatif $\delta a / a$ du paramètre $a$ pour une concentration $x$ de défauts est donné par :

$$
\frac{\delta a}{a x}=\frac{(1+\varepsilon) \chi n r_{0} F\left(r_{0}\right)}{9 \Omega}
$$

où $\Omega$ est le volume atomique $\Omega=\frac{4 \pi}{3} r_{a}^{3}$ et

$$
\varepsilon=\frac{r_{a}^{3}}{r_{0}^{3}} \chi\left(1+\frac{\chi^{\prime}-\chi}{3} \mu \chi^{\prime}\right),
$$

$\mu$ étant le coefficient de cisaillement de la matrice.

Le changement relatif $\delta \chi / \chi$ de la compressibilité pour une concentration $x$ de défauts est :

$\frac{\delta \chi}{\chi x}=\frac{r_{a}^{3}}{r_{0}^{3}}\left(1+\frac{4}{3} \mu \chi\right) \varepsilon+(1+\varepsilon)^{2} \frac{\chi n r_{0}^{2}}{9 \Omega} \frac{\mathrm{d} F\left(r_{0}\right)}{\mathrm{d} r_{0}}$.

Pour. le cas des impuretés, on peut prendre $\chi^{\prime}=\chi,(9)$ et (10) deviennent alors :

$$
\left\{\begin{array}{l}
\frac{\delta a}{a x}=\frac{\chi n r_{0}}{9 \Omega} F\left(r_{0}\right) \\
\frac{\delta \chi}{\chi x}=\frac{\chi n r_{0}^{2}}{9 \Omega} \frac{\mathrm{d} F\left(r_{0}\right)}{\mathrm{d} r_{0}} .
\end{array}\right.
$$

Pour le cas des lacunes, on peut prendre $\chi^{\prime}=\infty$.

Ce modèle est évidemment assez grossier. Il surestime quelque peu l'énergie élastique et néglige l'influence des défauts sur les ions autres que proches voisins.

3. Comparaison avec l'expérience. - Ainsi qu'il a été dit ci-dessus, les effets prévus doivent dépendre essentiellement de la valence de la matrice. La comparaison avec l'expérience sera donc faite en discutant les métaux d'après leur valence.

a) Métaux monovalents. - Dans le cas des métaux nobles (CFC) la figure 2 montre que l'interaction doit être répulsive si $\alpha_{1} \alpha_{2}>0$, les changements de paramètre cristallin et de compressibilité positits si $\alpha$ est positif. Ces résultat sont presque toujours observés.

Pour les interactions entre impuretés, on trouve théoriquement, pour des impuretés de différence de valence $z$ avec la matrice $: W \simeq z^{2} 0,03 \mathrm{eV}$ les expériences [7] donnent :

$$
\begin{array}{llll}
\text { Cu Zn } & 0,048 \mathrm{eV} & \text { Ag Cd } & 0,065 \mathrm{eV} \\
\text { Cu Ga } & 0,103 \mathrm{eV} & \text { Ag In } & 0,125 \mathrm{eV}
\end{array}
$$

On a pour les changements relatifs de paramètre divisés par $z$ :

$$
\begin{array}{lccc} 
& & \text { E`p. }\left[{ }^{\natural}\right] & \text { Th. } \\
\frac{\delta a}{a z} \quad \text { dans } & \text { ('u } & 0,04 & 0,02 \\
& \text { Ag } & 0,04 & 0,02
\end{array}
$$

et les changements de compressibilité :

$$
\begin{array}{lllll} 
& & \text { Exp. [9] } & \text { Th. } \\
\frac{\delta \chi}{\chi z} \quad \text { dans } & \mathrm{Cu} & 0,6 & 0,2 \\
& & \mathrm{Ag} & 0,45 & 0,2
\end{array}
$$

les résultats expérimentaux sont un peu dispersés dans le cas des effets de taille et très dispersés dans le cas des compressibilités.

Les effets de taille dans les alliages de métaux nobles entre eux peuvent être expliqués de la même manière, si on admet un changement de signe dans les pseudo-potentiels de $\mathrm{Cu}$ et $\mathrm{Ag} \mathrm{Au}$.

Expérimentalement, les interactions entre lacunes dans les métaux nobles semblent être attractives alors que le modèle théorique prévoit une répulsion. Ceci n'est pas très étonnant, car les lacunes sont de grosses perturbations et le modèle utilisé ici, en particulier pour des lacunes voisines, est certainement très imparfait. Il est certainement meilleur pour décrire les interactions entre lacunes et impuretés, de l'ordre de $-z 0,03 \mathrm{eV}$, ce qui est une valeur raisonnable [10]. Les lacunes contractent le réseau [11] $\delta a \mid a x \simeq 0,15$, alors que le modèle prévoit une contraction plus faible $\delta \alpha / a x \simeq 0,03$.

Les effets de taille dans les alliages entre métaux alcalins (cubiques centrés) sont assez bien expliqués.

$\begin{array}{lllll} & \text { Cs K } & \text { K Cs } & \text { Rb Cs } & \text { Cs Rb } \\ \text { Exp. [8] } & -0,14 & 0,13 & -0,07 & -\overline{0,075} \\ \text { Th. } & -0,06 & 0,075 & 0,04 & -0,04\end{array}$

On prévoit une contraction due aux lacunes plus importante que pour les métaux nobles $\delta \alpha / a x \simeq 0,1$. Expérimentalement, la contraction semble très importante.

b) Métaux divalents. - Pour un métal hexagonal compact, la figure 2 montre que l'interaction doit être attractive si $\alpha_{1} \alpha_{2}>0$, les effets de taille positifs pour $\alpha>0$. Les interactions doivent être de l'ordre de $-z^{2} 0,015 \mathrm{eV}$ - ce qui est en accord raisonnable avec l'expérience [7]

$(\mathrm{Zn} \mathrm{Ga}-0,025 \mathrm{eV}, \mathrm{Cd}$ In $-0,02 \mathrm{eV})$.

Pour un métal déviant appréciablement de la structure hexagonale compacte, il pourrait y avoir formation de zones linéaires par exemple.

Expérimentalement, les effets de taille sont moins clairs. Ils ont le même signe en général pour les paramètres $a$ et $c$ dans les alliages de $\mathrm{Mg}$, des signes opposés dans ceux de Zn. Ceci est compré- 
hensible car $\mathrm{Mg}$ a une structure presque exactement compacte. Au contaire, $\mathrm{Zn}$ dévie assez de la structure compacte. Pour les alliages de $\mathrm{Zn}$, le changement relatif de volume $\delta v / v=2 \delta \alpha / a+\delta c / c$ a le bon ordre de grandeur, mais dans un métal anisotrope, il peut être plus facile de comprimer suivant l'axe $c$ que perpendiculairement et obtenir des changements importants et opposés de $a$ et $c$ avec un changement de volume assez faible. Une étude plus détaillée serait certainement nécessaire.

c) Métaux trivalents. - Dans ce cas, la figure 2 montre que l'interaction entre impuretés doit être attractive et de l'ordre de $-z^{2} 0,01 \mathrm{eV}$, ce qui est en accord raisonnable avec l'expérience. Cette attraction donne-lieu à la formation des zones de Guinier-Preston dans les alliages $\mathrm{Al} \mathrm{Zn} ; \mathrm{Al} \mathrm{Ag}$, $\mathrm{Al} \mathrm{Cu}$, les zones sont sphériques pour les deux premiers alliages; pour le cas $\mathrm{Al} \mathrm{Cu}$, la formation de zones planes peut être expliquée par la superposition d'un effet de taille important aux interactions chimiques. Les effets de taille prévus doivent être négatifs pour $z>0$ ce qui est bien observé.

\begin{tabular}{llrr} 
& & Exp. [8] & \multicolumn{1}{c}{ Th. } \\
$\frac{\delta \alpha}{a x}$ & $\mathrm{Mg}$ & 0,06 & \multicolumn{0}{c}{} \\
& $\mathrm{Si}$ & $-0,035$ \\
& $-0,05$ & $-0,035$
\end{tabular}

La situation est moins claire en ce qui concerne les impuretés de période différente. Il est à remarquer que le signe de ces effets est opposé à celui des métaux nobles, ce qui est expérimentalement observé.

Les interactions entre lacunes et impuretés doivent être de l'ordre de $\alpha 0,035 \mathrm{eV}$, les lacunes doivent donner lieu à un effet de taille positif.

En conclusion, le modèle proposé explique qualitativement et semi-quantitativement les interactions entre défauts et les changements de paramètres et de compressibilités dus aux défauts. II explique, en particulier, l'observation d'effets de signes opposés dans les métaux de valence différente et permet de relier entre eux divers phénomènes et de les comprendre d'une manière cohérente. Ces effets sont dus aux oscillations de densité électronique dans la bande de conduction autour de chaque défaut.

\section{RÉFÉRENCES}

[1] Friedel (J.), Phil. Mag., 1952, 43, 153. Fiuderman (M. A.) et Kittel (C.), Phys. Res., 1954, 96, 99.

[2] Langer (J. S.) et Vosko (S. H.), J. Phys. Chem. of Solids, 1960, 12, 196.

[3] Rотн (L.), Thèse, Harvard University, 1957. Blandin (A.), J. Physique Rad., 1961, 22, 507.

[4] Kонn (W.) et Vosko (S. H.), Phys. Rev., 1960, 119, 912.

Blandin (A.) et Friedel (J.), J. Phys. Rad., 1960, 21, 689.

[5] Ham (F. S.), The Fermi Surface, John Wiley and Sons, 1960 .
[6] Corless (G. K.) et March (N. M.), Phll. Mag., 1961, $6,1285$.

[7] KLEPPA (O. J.), Colloque sur les solutions solides, Orsay, 1962.

[8] Pearson (W. B.), A. Handbook of lattice spacings and structures of metals and alloys, Pergamon Press, 1958.

[9] Bacon (R.) et Smith (C. S.), Acta Met., 1956, 4, 336. Neighbours (J. R.) et Smith (C. S.), Acta Met., 1954, 2, 591 .

[10] Fly n (C. P.), Phys. Rev., 1962, 125, 881.

[11] Simmons (R. O.) et Balluffi (R. W.), Phys. Reo., $1962,125,862$. 